\title{
The effect of tumor locations of esophageal cancer on the metastasis to liver or lung
}

\author{
Dashan $\mathrm{Ai}^{1,2}$, Yun Chen ${ }^{1,2}$, Qi Liu ${ }^{1,2}$, Jiaying Deng ${ }^{1,2}$, Kuaile Zhao ${ }^{1,2}$ \\ ${ }^{1}$ Department of Radiation Oncology, Fudan University Shanghai Cancer Center, Shanghai 200032, China; ${ }^{2}$ Department of Oncology, Shanghai \\ Medical College, Fudan University, Shanghai 200032, China \\ Contributions: (I) Conception and design: D Ai, Y Chen, K Zhao; (II) Administrative support: K Zhao; (III) Provision of study materials or patients: D \\ Ai, Y Chen, Q Liu; (IV) Collection and assembly of data: D Ai, J Deng; (V) Data analysis and interpretation: D Ai, K Zhao; (VI) Manuscript writing: \\ All authors; (VII) Final approval of manuscript: All authors. \\ Correspondence to: Kuaile Zhao. Professor, Department of Radiation Oncology, Fudan University Shanghai Cancer Center, 270 Dong'An Road, \\ Shanghai 200032, China. Email: kuaile_z@sina.com.
}

Background: Liver and lung are the most common metastatic sites for esophageal cancer. Tumor location of esophageal cancer has been a prognostic factor of tumor, node and metastasis (TNM) staging system since 2009. However, the relationship between tumor location and metastatic sites remains unclear.

Methods: Patients with esophageal cancer were derived from surveillance, epidemiology and end results (SEER) database from 2010 to 2013. Age, sex, histologic type and grade were included in the model for propensity score matching between patients with or without liver and lung metastasis. After propensity score matching, the relationship between tumor location and the probability of liver or lung metastasis was analyzed using chi-square analysis. Overall survival was analyzed between different locations within liver or lung metastasis patients.

Results: A total of 6,812 patients were eligible. After matching, primary site was still an independent risk factor for both liver and lung metastasis. For patients with liver metastasis, lower esophagus was more likely to be the primary site than upper segment $(\mathrm{P}=0.014)$. In contrast, lung metastasis was more likely to be found in patients with upper esophageal cancer than lower segment $(\mathrm{P}=0.033)$. In liver metastasis group, those with upper esophageal cancer had the worst survival, followed by middle and lower segment and the results are opposite in lung metastasis group.

Conclusions: These findings are useful in the evaluation when diagnose and follow up. Targeted examinations in accordance with tumor location will assist to find early metastasis and deliver treatment timely and properly.

Keywords: Esophageal cancer; tumor location; liver metastasis; lung metastasis

Submitted Jul 01, 2019. Accepted for publication Sep 12, 2019.

doi: $10.21037 /$ jtd.2019.09.67

View this article at: http://dx.doi.org/10.21037/jtd.2019.09.67

\section{Introduction}

Esophageal cancer is the sixth leading cause of cancerrelated deaths worldwide, with 455,800 new cases and 400,200 deaths estimated in 2012 (1). In UICC/AJCC staging system (2), tumor location was first brought into $7^{\text {th }}$ edition staging system for esophageal cancer, which indicated that tumor location could affect the prognosis. Dismal prognosis was seen in esophageal cancer and prognosis of patients with metastatic disease was much poorer. In accordance with the results from autopsy (3), lung and liver were the most common metastatic sites, which are being affected by $31 \%$ and $23 \%$ of the patients, respectively. In our previous study, liver and lung metastasis were shown most frequently in patients with esophageal cancer (4). However, there was no study, 


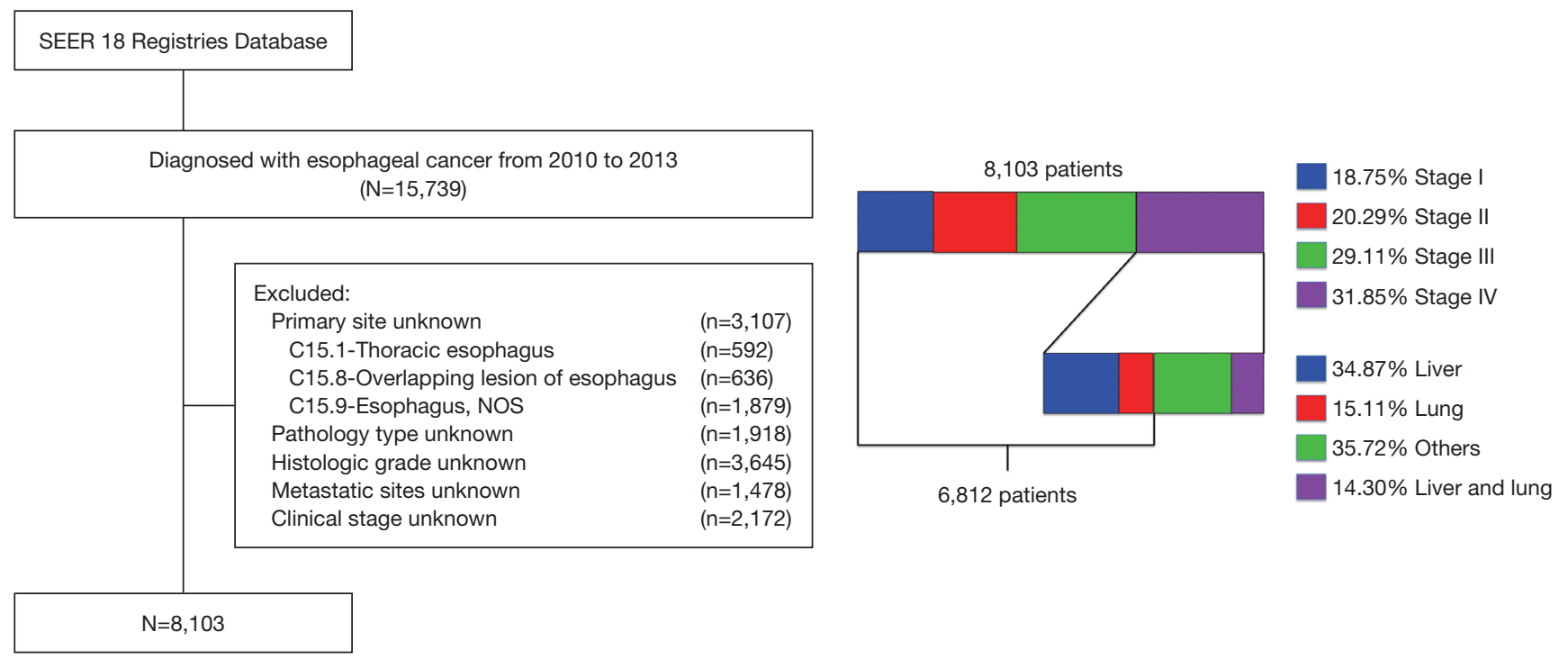

Figure 1 Flow chart of patient selection from SEER database (2010-2013). SEER, surveillance, epidemiology and end results; NOS, not otherwise specified.

which systematically described the characteristics of liver and lung metastasis in esophageal cancer, especially the relationship between different primary sites and metastasis to liver or lung.

In this study, we analyzed the relationship between different tumor locations of esophageal cancer and metastasis to liver or lung using surveillance, epidemiology and end results (SEER) database.

\section{Methods}

In this study, patients included $(\mathrm{n}=15,739)$ were diagnosed esophageal cancer from 2010 to 2013 . We excluded a total of 8,927 patients mainly because of unknown primary site $(n=3,107)$, pathology type $(n=1,918)$, histologic grade $(n=3,645)$, metastatic site $(n=1,478)$, or clinical stage $(\mathrm{n}=2,172)$. We also excluded stage IV patients without liver or lung metastasis $(\mathrm{n}=922)$ or both liver and lung metastasis $(n=369)$. A total of 6,812 patients with esophageal cancer matching the specified criteria were included in the final sample for this analysis (Figure 1).

In this study, upper esophagus was defined with primary site codes C15.0 (cervical esophagus) and C15.3 (upper third of esophagus). Code C15.4 (middle third of esophagus) was used to identify the middle esophagus. Lower esophagus was a combination of codes C15.2 (abdominal esophagus) and C15.5 (lower third of esophagus). Histologic codes
8140-8389 were used to define adenocarcinoma and codes 8050-8089 for squamous cell carcinoma.

In this study, propensity score based matching analysis was performed between patients with or without liver and lung metastasis to reduce the effect of potential selection bias in this retrospective study. Independent variables entered into the propensity model included age, sex, histologic type and grade. A 1:1 matching between the groups was accomplished by using the nearest-neighbor matching method with a caliper distance of 0.01 without replacement. After matching, the relationship between tumor location and liver or lung metastasis was analyzed using chi-square analysis.

Overall survival was defined as the period from diagnosis to death as a result of any cause. Survival estimation and comparison among different variables were performed using Kaplan-Meier method.

A two-sided $\mathrm{P}$ value of $<0.05$ was considered statistically significant. All statistical analyses were performed using SPSS 19.0 (SPSS, Chicago, IL, USA), and the survival curves were drawn with GraphPad Prism 6.0 (GraphPad Software, San Diego, CA, USA).

\section{Results}

The study group consisted of 6,812 patients with esophageal cancer diagnosed from 2010 to 2013, including 
Table 1 Demographic and clinical characteristics of patients with and without liver or lung metastases before and after matching

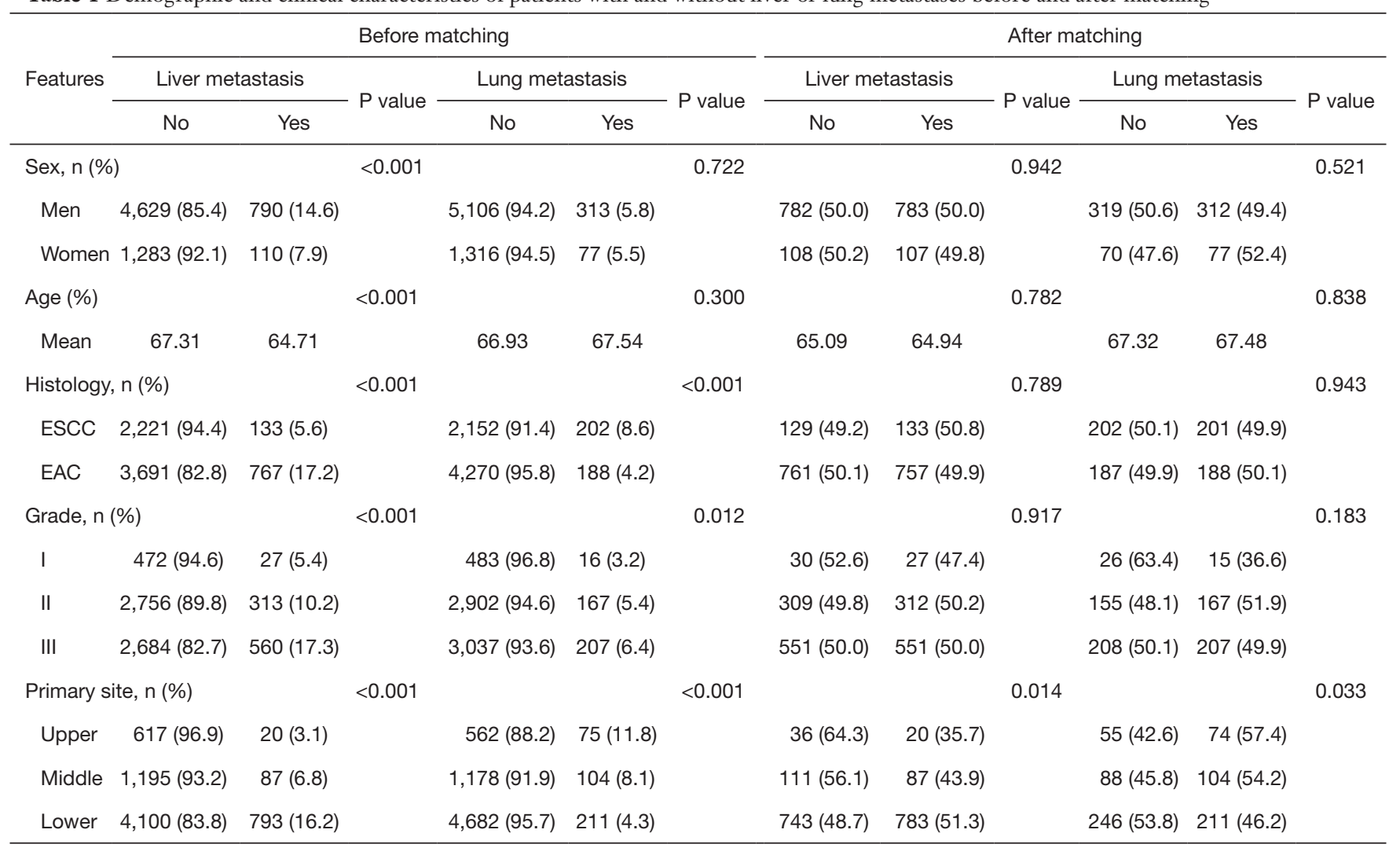

ESCC, esophageal squamous cell carcinoma; EAC, esophageal adenocarcinoma.

1,393 women (20.4\%) and 5,419 men (79.6\%). Median age was 67 and around two-thirds of patients were adenocarcinoma. Disease-related data showed that $71.8 \%$ patients were diagnosed with lower esophageal cancer, while patients with middle esophageal cancer accounted for $18.8 \%$ and upper esophageal cancer $9.4 \%$. Only $7.3 \%$ tumors were well differentiated (grade I), while $45.1 \%$ were moderately differentiated (grade II) and $47.6 \%$ were poorly differentiated or undifferentiated (grade III or IV). According to the database, there were 900 patients (13.2\% among all-stage patients and $34.9 \%$ in stage IV patients) with liver metastasis only and 390 (5.7\% among all-stage patients and $15.1 \%$ in stage IV patients) with lung metastasis only.

In the initial data before matching, there were significant differences in sex, age, primary site, histologic type and grade between patients with and without metastasis. Patients with liver metastasis were significantly younger, more likely to be men and had a higher percentage of adenocarcinoma, while patients with lung metastasis had a higher probability of squamous cell carcinoma and poor differentiation (Table 1).

After matching, there were no significant differences in sex, age, histologic type and grade between patients with and without liver or lung metastasis. At the same time, primary site was still an independent risk factor for both liver and lung metastasis $(\mathrm{P}=0.014$ for liver metastasis and $\mathrm{P}=0.033$ for lung metastasis) (Table 1). For patients with liver metastasis, lower esophagus was more likely to be the primary site than upper segment. In contrast, lung metastasis was more likely to be found in patients with upper esophageal cancer rather than lower segment.

Survival curves for patients with liver or lung metastasis were shown in Figure 2. In liver metastasis group, those with upper esophageal cancer had the worst survival, followed by middle and lower segment [Figure $2 A$, median survival time, 2 months (upper), 4 months (middle) and 6 months (lower), $\mathrm{P}=0.003]$. In contrast, patients with upper esophageal cancer had a better trend in overall survival than lower and middle segment in lung metastasis group, even though no statistical difference was detected [Figure 2B, median survival time, 

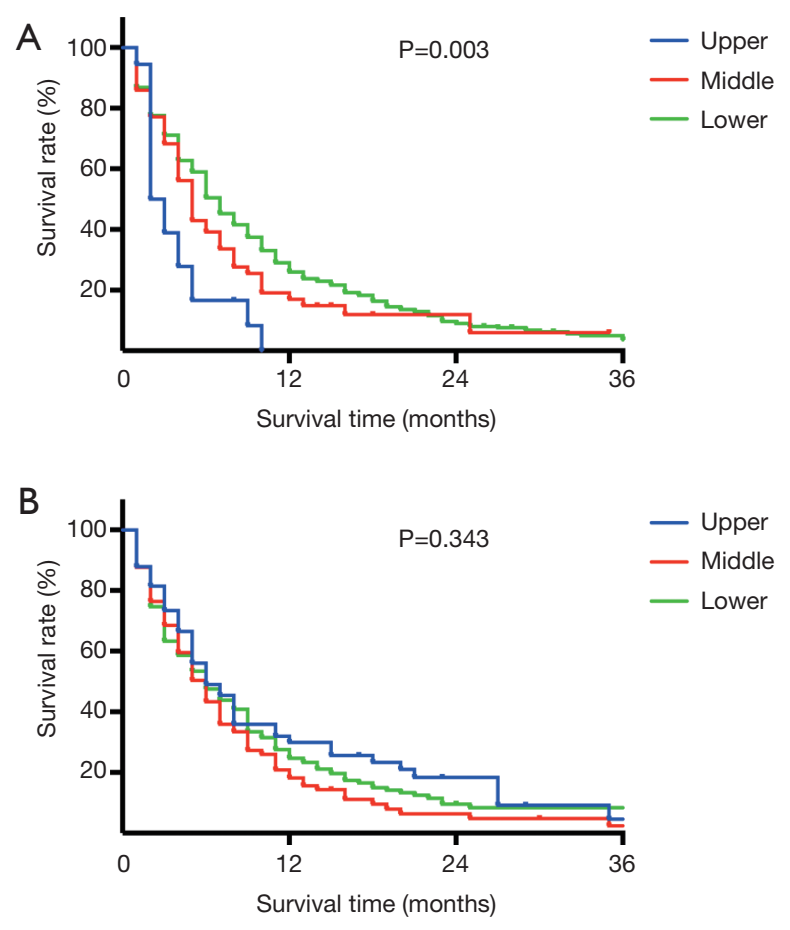

Figure 2 Survival curves of overall survival by tumor location (A) in patients with liver metastasis $(\mathrm{P}=0.003),(\mathrm{B})$ in patients with lung metastasis $(\mathrm{P}=0.343)$.

6 months (upper), 5 months (middle) and 5 months (lower), $\mathrm{P}=0.343]$.

\section{Discussion}

In this study, we analyzed the different impact of tumor location of esophageal cancer on liver or lung metastasis. Upper esophageal cancer was more relevant to lung metastasis while lower esophageal cancer to liver metastasis.

Liver and lung are two of the most common metastatic organs in most gastrointestinal carcinoma, which may possibly be due to the rich blood supply and the interaction through vascular system between different organs. Traditional hypothesis, including anatomical/mechanical hypothesis (5) are widely accepted in the explanation of metastatic regulations (Figure 3).

In the anatomical hypothesis, the vascular system in different segments may partly be responsible for the metastasis to specific organs and the first draining sites was exactly the most frequent metastatic site in our study. From the upper and middle part of the esophagus, blood is drained through inferior thyroid vein and azygos vein,

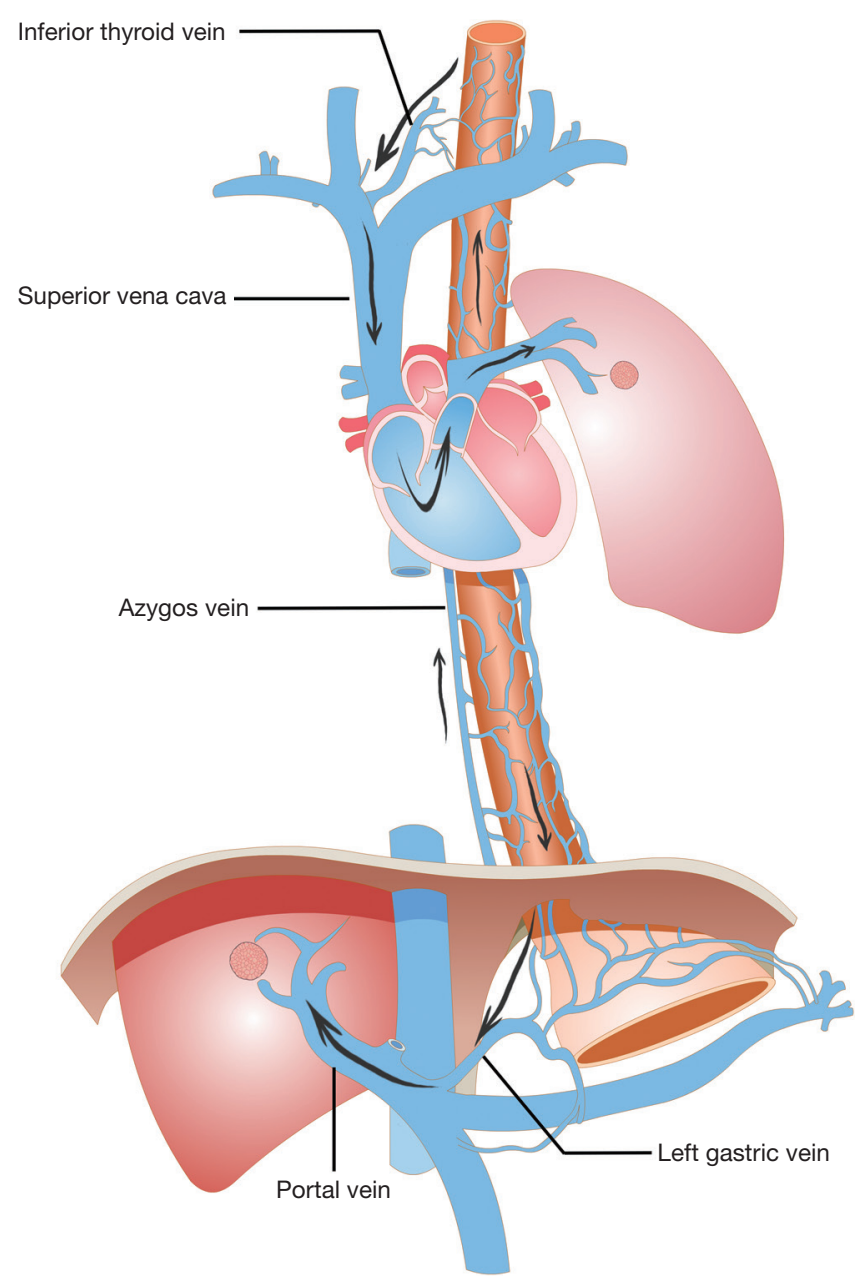

Figure 3 Metastatic mechanism to liver and lung. From the upper and middle part of the esophagus, blood with tumor cells is drained through inferior thyroid vein and azygos vein, which are branches of superior vena cava, and through heart and pulmonary circulation, the first draining site is lung. Through left gastric vein and portal system, the first drainage site from lower segment of esophagus is liver.

which are branches of superior vena cava, and through heart and pulmonary circulation, the first draining site is lung. Similarly, the first draining site of lower part of esophagus is liver through left gastric vein and portal system. Actually, due to intraparietal veins and other metastatic approaches, the changes in metastatic pattern from upper to lower segment are smooth, rather than "all or none", but the trend is still notable.

Through the survival analysis, metastases in same organ but from different primary tumor locations lead 
to different prognosis. According to the hypothesis above, liver metastasis is "more distant" for patients with upper esophageal cancer than those with middle or lower esophageal cancer. In contrast, for patients with upper esophageal cancer, lung metastasis is "less distant" due to anatomic structure. Metastases in more distant organs make worse survival and survival data provide some evidence for these hypotheses. In our results, patients with upper esophageal cancer had the worst survival in liver metastasis group and best survival in lung metastasis group than middle or lower segment, which is consistent with our hypothesis.

Similar anatomic structure makes similar metastatic regulations. Like esophageal cancer, the mechanical hypothesis could also be used in the explanation of metastasis from colorectal cancer (6). From the colon and proximal parts of the rectum, blood is drained through the portal system to the liver. The first drainage organ of the distal parts of rectum is the lung. Therefore, it seems logical that more metastasis from rectal cancer to lung and more from colon cancer to liver. The anatomical hypothesis could be part of explanation that different tumor locations make different metastatic sites.

Knowledge of metastatic patterns is useful in making clinical decisions, including early diagnosis and treatment. Evaluation when diagnosis should be systemic and targeted according to risk factors for specific site of metastasis. Early diagnose for metastasis could provide a chance for further treatment, including stereotactic body radiotherapy (SBRT), radiofrequency ablation and surgery when oligometastases, even though not for the purpose of cure, so as to prolong survival time and improve quality of life (7-11).

To our knowledge, this is the first SEER based study focusing on the relation between tumor location and liver and lung metastasis of esophageal cancer. However, there are still some limitations. First of all, due to the structure of database, we can only analyze data from 2010 to 2013, which is so limited. Secondly, this study did not consist of long-time follow up and the effects of different therapies. Moreover, most of the explanations were theoretical and anatomical/mechanical hypothesis helped to illustrate these phenomena. Further researches were needed to confirm these hypotheses.

Despite those limitations, specific esophageal tumor locations show a preference to specific target organs of metastasis. Upper esophageal cancer was more relevant to lung metastasis while lower esophageal cancer to liver metastasis. These findings are useful in the evaluation when diagnose and follow up, especially for those without metastasis. Targeted examinations in accordance with tumor location will assist to find early metastasis and deliver treatment timely and properly.

\section{Acknowledgments}

Funding: This study was supported by the National Natural Science Foundation of China (Grant 21172043, 81703160).

\section{Footnote}

Conflicts of Interest: The authors have no conflicts of interest to declare.

Ethical Statement: The authors are accountable for all aspects of the work in ensuring that questions related to the accuracy or integrity of any part of the work are appropriately investigated and resolved. In this study, we use data from surveillance, epidemiology and end results (SEER) database: a publicly available database. In our point of view, no ethics approval is needed.

\section{References}

1. Torre LA, Bray F, Siegel RL, et al. Global cancer statistics, 2012. CA Cancer J Clin 2015;65:87-108.

2. Rice TW, Blackstone EH, Rusch VW. 7th edition of the AJCC cancer staging manual: esophagus and esophagogastric junction. Ann Surg Oncol 2010;17:1721-4.

3. Mandard AM, Chasle J, Marnay J, et al. Autopsy findings in 111 cases of esophageal cancer. Cancer 1981;48:329-35.

4. Ai D, Zhu H, Ren W, et al. Patterns of distant organ metastases in esophageal cancer: a population-based study. J Thorac Dis 2017;9:3023-30.

5. Weiss L, Grundmann E, Torhorst J, et al. Haematogenous metastatic patterns in colonic carcinoma: an analysis of 1541 necropsies. J Pathol 1986;150:195-203.

6. Riihimäki M, Hemminki A, Sundquist J, et al. Patterns of metastasis in colon and rectal cancer. Sci Rep 2016;6:29765.

7. Lo SS, Moffatt-Bruce SD, Dawson LA, et al. The role of local therapy in the management of lung and liver oligometastases. Nat Rev Clin Oncol 2011;8:405-16.

8. Huddy JR, Thomas RL, Worthington TR, et al. Liver metastases from esophageal carcinoma: is there a role for surgical resection? Dis Esophagus 2015;28:483-7. 
9. Ichida $\mathrm{H}$, Imamura $\mathrm{H}$, Yoshimoto J, et al. Pattern of postoperative recurrence and hepatic and/or pulmonary resection for liver and/or lung metastases from esophageal carcinoma. World J Surg 2013;37:398-407.

10. Iitaka D, Shiozaki A, Fujiwara H, et al. Case involving long-term survival after esophageal cancer with liver and lung metastases treated by multidisciplinary therapy: report of a case. Surg Today 2013;43:556-61.

11. Milano MT, Katz AW, Zhang H, et al. Oligometastases treated with stereotactic body radiotherapy: long-term follow-up of prospective study. Int J Radiat Oncol Biol Phys 2012;83:878-86.
Cite this article as: Ai D, Chen Y, Liu Q, Deng J, Zhao K. The effect of tumor locations of esophageal cancer on the metastasis to liver or lung. J Thorac Dis 2019;11(10):4205-4210. doi: $10.21037 /$ jtd.2019.09.67 